\title{
TERAHERTZ PHOTONICS OF MICROPLASMA AND BEYOND
}

\author{
X.-C. Zhang ${ }^{a, b}$ and F. Buccheri ${ }^{a}$ \\ ${ }^{a}$ The Institute of Optics, University of Rochester, Rochester, NY 14627, USA \\ ${ }^{\mathrm{b}}$ The Beijing Advanced Innovation Center for Imaging Technology, Capital Normal University, Beijing 100037, China \\ Email: xi-cheng.zhang@rochester.edu
}

Received 18 January 2018; accepted 22 March 2018

\begin{abstract}
THz air photonics using laser-induced air plasma is one of the leading frontiers in the THz community. Ambient air, when excited with an intense femtosecond laser beam, exhibits the remarkable ability to generate and detect pulsed $\mathrm{THz}$ waves through a nonlinear optical process. Significant advances in the use of air plasma for emitting, controlling, enhancing and measuring broadband $\mathrm{THz}$ waves have opened up a range of research opportunities. However, one of the major challenges for the research community and in real world applications is that plasma formation requires the use of an intense laser (mJ pulse energy), but most femtosecond laser oscillators only have pulse energies in the range of $\mathrm{pJ}$ to tens of nJ. The investigation of THz photonics, specifically the exploration of laserinduced plasmas at the micro-nano scale and beyond, is a frontier. Microplasmas generated by tightly focused optical excitation beams with controlled polarization serve as a new $\mathrm{THz}$ source with its unique radiation pattern and easy operation. The laser energy threshold for $\mathrm{THz}$ wave generation, the power scaling and the generation efficiency from microplasmas are significantly different from those of elongated plasmas. Our estimation indicates that the micronano plasma approach could reduce the necessary optical pulse energy by five orders of magnitude, while still obtaining a comparable or better signal-to-noise ratio for THz time-domain spectroscopy. This would be made possible by the high electron density $\left(10^{19} \mathrm{~cm}^{-3}\right.$ or more $)$ achievable with a tight focus laser excitation, which correlates with the $\mathrm{THz}$ generation efficiency, and the use of laser oscillators with a much higher pulse repetition rate, as compared to the currently employed amplified laser systems $(100 \mathrm{MHz}$ vs $1 \mathrm{kHz})$. The THz micro-nano plasma is expected to lead to key technologies that will enable further interdisciplinary research and continued advancements of numerous $\mathrm{THz}$ wave sensing and spectroscopy developments. It serves as a vehicle for studying the extreme $\mathrm{THz}$ science.
\end{abstract}

Keywords: ultrafast optical phenomena, infrared, submillimetre wave, microwave and radiowave sources, ultrafast spectroscopy

PACS: 07.57.Hm, 78.47.J-

\section{THz air photonics}

Since the $\mathrm{THz}$ generation process in air plasma is intimately tied with gas ionization, it requires intensities greater than $10^{12} \mathrm{~W} / \mathrm{cm}^{2}$ to be created near the target location. While this can be achieved through geometric focusing with lenses or curved mirrors, it can also be accomplished with a technique known as filamentation [1] mentation is the process by which a high-intensity beam self-focuses through nonlinear processes and collapses, forming a plasma channel with $\mathrm{mm}$ to $\mathrm{cm}$ lengths. This plasma channel stabilizes the beam at very small diameters ( 30 to $100 \mu \mathrm{m}$ ) and maintains high intensities over ranges much longer than the Rayleigh length of a traditional, geometrically focused beam.

Figure 1(a) illustrates this all-optical process for the generation and detection of $\mathrm{THz}$ waves using an air emitter and sensor, which we call $\mathrm{THz}$ air-biased-coherent-detection (THz-ABCD). A Ti:sapphire regenerative amplifier $(\sim 3 \mathrm{~mJ}$, $30 \mathrm{fs}$ and $1 \mathrm{kHz}$ repetition rate with a central wavelength at $800 \mathrm{~nm}$ ) is used as a laser source. The laser beam is focused through a $100-\mu \mathrm{m}$ thick type-I beta barium borate (BBO) crystal to generate its second harmonic beam. The mixed fundamental $(\omega)$ and its second harmonic $(2 \omega)$ beams 
(a)



(b)

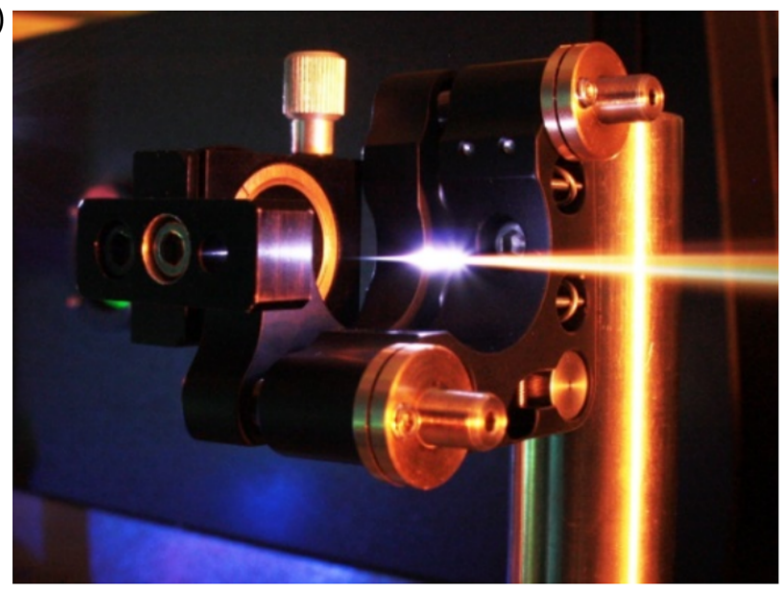

generate a $\mathrm{THz}$ wave at the ionizing plasma spot. The $\mathrm{THz}$ beam is recombined with the fundamental beam at a second focus, creating another plasma spot, which serves as the detection region. An electric bias field is applied to the detection region, supplying a second harmonic local oscillator for coherent detection via a photomultiplier detector [2]. The plasma emits a very intense, highly directional, ultra-broadband $\mathrm{THz}$ field with its temporal signal, as shown in Fig. 1(b).

Similar to the widely used generation and detection of $\mathrm{THz}$ waves in electro-optic crystals by second-order optical nonlinearity, the $\mathrm{THz}$ waves can be detected by the third-order optical nonlinearity in air. This $\mathrm{THz}$ wave air-plasma photonics system [3, 4], which differentiates itself from most $\mathrm{THz}$ time-domain spectroscopy systems [5-10], uses ambient air or selected gases for both the generation and the detection of broadband $\mathrm{THz}$ waves.

Figures 2(a, b) plot a typical temporal $\mathrm{THz}$ waveform and its spectrum from laser-induced air plasma with the peak $\mathrm{THz}$ electric field exceeding $1 \mathrm{MV} / \mathrm{cm}$, using $\mathrm{THz}$ air-biased coherent detection (THz-ABCD), which is explained in the following section. The dynamic range is over 3000
Fig. 1. (a) A schematic diagram of the THz$\mathrm{ABCD}$ design concept. A laser pulse is focused through a beta barium borate $(\mathrm{BBO})$ crystal to generate its second harmonic beam. The mixed fundamental and its second harmonic beams generate a $\mathrm{THz}$ wave at the ionizing plasma spot. The $\mathrm{THz}$ and optical beams are recombined at the detection region. An electric bias (HV) is applied to the detection region, supplying a second harmonic local oscillator for coherent detection. (b) Laser-induced air plasma (central bright line) emits an intense THz field.


Fig. 2. (a) The time-resolved $\mathrm{THz}$ signal generated and detected using the ABCD method in dry nitrogen gas. (b) The log scale plot of the spectrum. 
and its bandwidth covers the $\mathrm{THz}$ gap $(0.3 \mathrm{THz}$ to $10 \mathrm{THz}$ ). THz-ABCD has now become a standard $\mathrm{THz}$ wave detection technique for researchers involved in projects investigating ultra-broadband $\mathrm{THz}$ pulses and their applications.

\section{THz air-biased coherent detection (ABCD)}

The basic detection principle utilizes gas to sense the pulsed $\mathrm{THz}$ waves by measuring the $\mathrm{THz}-$ field-induced optical second harmonic generation through a third-order nonlinear process [11, 12]. Here, by focusing the $\mathrm{THz}$ pulse with the probe beam fundamental $(\omega)$ pulse, an optical field at the second harmonic frequency $(2 \omega)$ is formed such that [13]

$$
E_{2 \omega}(t) \propto \chi^{(3)} E_{\mathrm{THz}}(t-\tau) E_{\omega}(t) E_{\omega}(t) E_{2 \omega}^{\mathrm{LO}},
$$

where $E_{2 \omega}, E_{\omega}(t)$, and $E_{\mathrm{THz}}$ are the electric field amplitudes of the $2 \omega, \omega$, and $\mathrm{THz}$ waves, respectively, and $\chi^{(3)}$ is the third-order susceptibility of the gas.

Equation (1) shows that the intensity of the measured second-harmonic signal by the photon detector is proportional to the intensity of the $\mathrm{THz}$ wave, or $I_{2 \omega}(t) \propto I_{\mathrm{THz}}(t-\tau)$. Unfortunately, this measurement of $I_{\mathrm{THz}}$ is incoherent, and the phase information is lost, limiting its usefulness in applications [ [4].

However, we can perform $\mathrm{THz}$ time-domain spectroscopy to achieve coherent detection. By introducing an external bias to the optical focus, a bias-field-induced second harmonic pulse with the field amplitude designated by $E_{2 \omega}^{\mathrm{LO}}$ is generated, and interferes with the THz-field-induced second harmonic pulse $E_{2 \omega}^{\mathrm{THz}}$. Alternating the direction of the bias field adjusts the carrier-envelope phase relative to that of the $\mathrm{THz}$-field-induced second harmonic pulse from 0 to $\pi$, giving a second-harmonic intensity of the form

$$
\begin{aligned}
& I_{2 \omega}(t) \propto\left\langle E_{2 \omega}^{2}\right\rangle=\left\langle\left(E_{2 \omega}^{\mathrm{THz}}+E_{2 \omega}^{\mathrm{LO}}\right)^{2}\right\rangle \\
& =\left\langle\left(E_{2 \omega}^{\mathrm{THz}}\right)^{2}\right\rangle \pm 2\left\langle E_{2 \omega}^{\mathrm{THz}} E_{2 \omega}^{\mathrm{LO}}\right\rangle+\left\langle\left(E_{2 \omega}^{\mathrm{LO}}\right)^{2}\right\rangle .
\end{aligned}
$$

We can drop the time-averaging notation, as the cross-term has a constant value (but alternating sign) for a square-wave bias field. Expressing $E_{2 \omega}^{\mathrm{THz}}$ and $E_{2 \omega}^{\mathrm{LO}}$ as in Eq. (1), Eq. (2) can be written as

$$
\begin{aligned}
& I_{2 \omega}(t) \propto\left(\chi^{(3)} I_{\omega}(t)\right)^{2}\left[\left(E_{\mathrm{THz}}(t-\tau)\right)^{2}\right. \\
& \left. \pm 2 E_{\text {bias }} E_{\mathrm{THz}}(t-\tau)+\left(E_{\text {bias }}\right)^{2}\right] .
\end{aligned}
$$

Only the cross term is present at the modulation frequency, while the other terms exist only at the laser repetition rate and its even harmonics. Thus lock-in amplification easily isolates the heterodyne signal proportional to $E_{\mathrm{THz}}$. The measured second harmonic intensity at the modulation frequency will be

$$
I_{2 \omega}(\tau) \propto 2\left[\chi^{(3)} I_{\omega}(t)\right]^{2} E_{\text {bias }} E_{\mathrm{THz}}(t-\tau) .
$$

The linearity of $I_{2 \omega}$ versus $E_{\text {bias }}$ indicates heterodyne detection when $E_{\text {bias }}$ is treated as a local oscillator.

To test the validity of Eq. (4), Fig. 3(a) schematically illustrates the concept. $E_{\mathrm{dc}}$ acts as the local oscillator $E_{\text {bias }}$. Figure 3(b) plots the detected $\mathrm{THz}$ field versus $\chi^{(3)}$, normalized by the value of nitrogen $\chi^{(3)}\left(\mathrm{N}_{2}\right)$ in six saturated alkane $\left(\mathrm{C}_{n} \mathrm{H}_{2 n+2}\right)$ gases. Using $\mathrm{C}_{6} \mathrm{H}_{14}$ the detection mechanism is 24 times more sensitive compared to using $\mathrm{N}_{2}$ or air. The signal-to-noise ratio (SNR) increases with increased $I_{\omega}(t)$ until the probe energy reaches the plasma threshold, as shown in Fig. 3(c). Figure $3(\mathrm{~d})$ shows the $\mathrm{THz}$ field versus the bias DC field in air, $\mathrm{Xe}$ and $\mathrm{SF}_{6}$ under the same pressure.

Equation (4) could be the key to optimizing $\mathrm{THz}$ wave detection in gases. Equation (4) shows that the field-induced-second-harmonic signal $I_{2 \omega}$ is quadratically proportional to $\chi^{(3)}$ and $I_{\omega}(t)$. Therefore, using gases with a large $\chi^{(3)}$ or at higher gas pressure (since the effective $\chi^{(3)}$ is proportional to the number of molecules at low pressure) or with higher probe pulse energy are the best choices. We will test conjugated hydrocarbons, which contain alternating single and double bonds. These conjugated hydrocarbons (such as $\mathrm{C}_{4} \mathrm{H}_{6}$ and $\mathrm{C}_{5} \mathrm{H}_{8}$ ) can possess an extremely large nonlinear optical response due to their $\pi$ bonds, which tend to be delocalized and respond more freely to an applied optical field.

\section{Microplasmas}

The widespread use of $\mathrm{THz}$ air photonics has been limited by the amount of laser energy required to implement the technique. We address this 


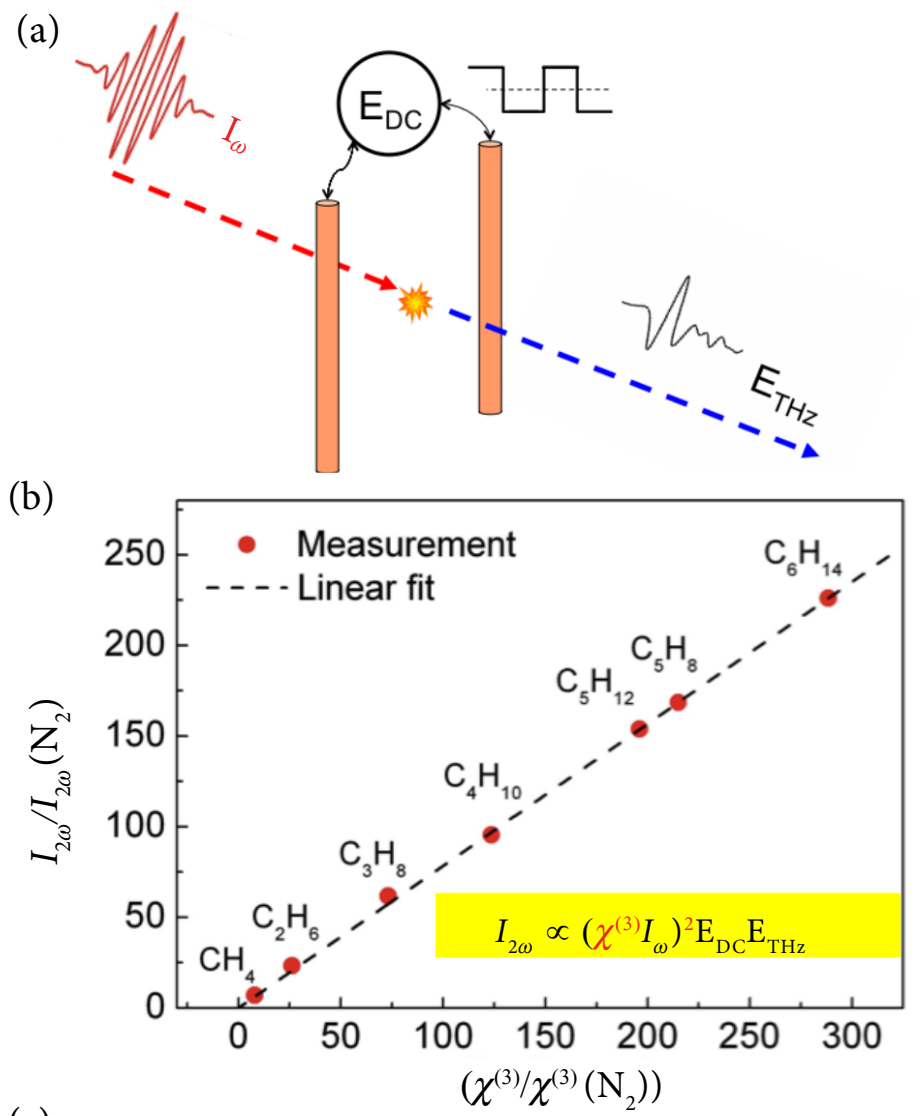

(c)

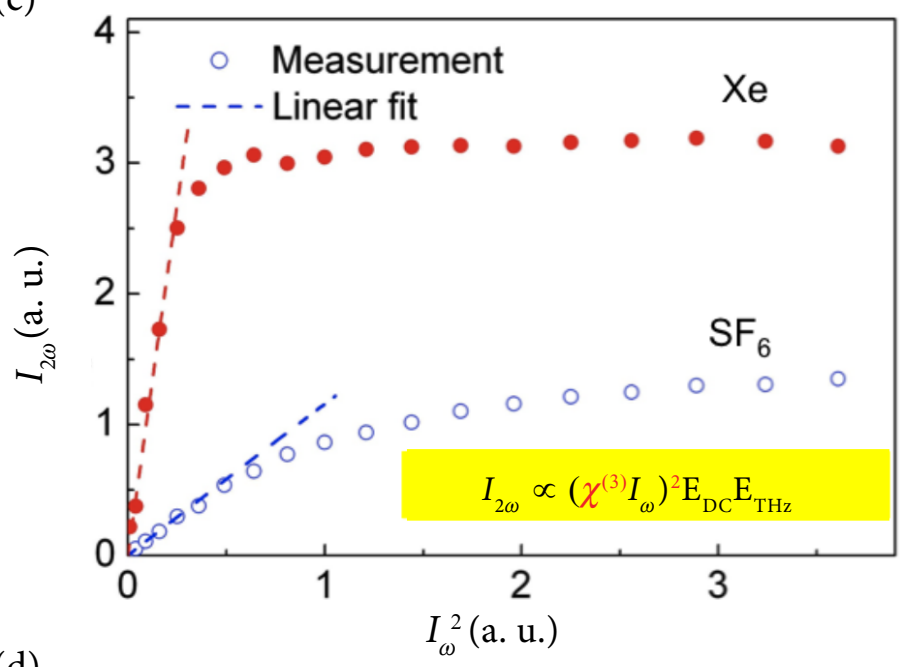

(d)

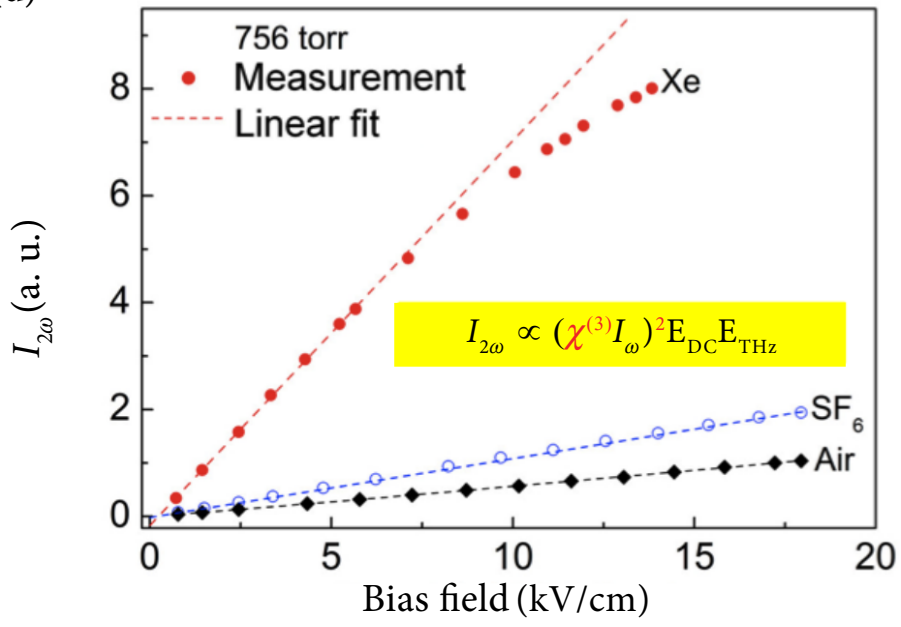

Fig. 3. (a) The heterodyne concept of THz-ABCD. (b) The measured THz field versus normalized $\chi^{(3)}$ from saturated alkane $\left(\mathrm{C}_{n} \mathrm{H}_{2 n+2}\right)$ gases. $\mathrm{C}_{6} \mathrm{H}_{14}$ as a sensor is 243 times more sensitive than $\mathrm{N}_{2}$ or air. (c) The plots of dependence at low power (under the plasma threshold) indicate validation of the four-wave-mixing theory. (d) The plots of $E_{\mathrm{THz}}$ versus $E_{\text {bias }}$ in air, $\mathrm{SF}_{6}$, and Xe. 
problem by proposing to replace the typical filament currently used (length of several $\mathrm{mm}$ to $\mathrm{cm}$ ) with a sub-mm plasma, which we call a "microplasma". A microplasma can be created by focusing a laser beam in ambient air through a high numerical aperture (NA) objective. The energy required to generate $\mathrm{THz}$ waves from such microplasma can be many orders of magnitude smaller compared to that of a filament.

Figure 4 (a) shows the concept of $\mathrm{THz}$ emission (drawn as blue (online) pulses) from a microplasma. The $\mathrm{THz}$ emission direction is nearly perpendicular with respect to the optical axis; therefore it is spatially separated from the residual laser excitation. We attribute this sideways $\mathrm{THz}$ radiation

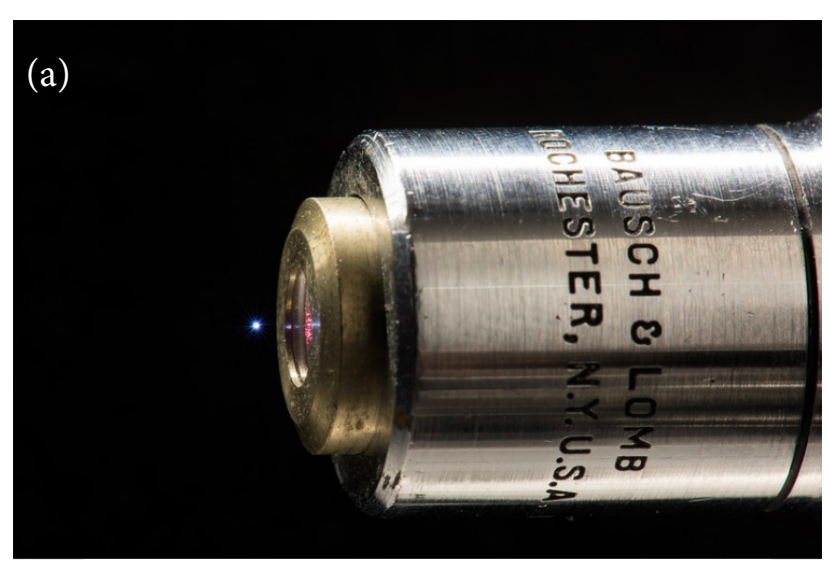

(b)

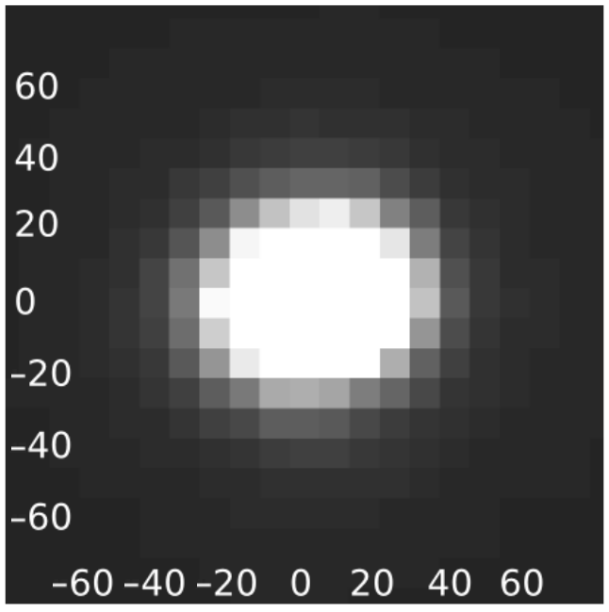

Fig. 4. (a) The concept of $\mathrm{THz}$ generation from laserinduced microplasma. The THz signal is spatially separated from the residual laser excitation. (b, c) Photos of the microplasma fluorescence obtained by focusing the laser beam with a high NA microscope objective. The pictures were taken with an iCCD camera. (c) A magnified image of the microplasma (unit is $\mu \mathrm{m}$ ). However, the plasma spot contribution to the $\mathrm{THz}$ wave is about one $\mu \mathrm{m}$ or less. to the steep ponderomotive potential present at the focal plane, which accelerates the free electrons created by photoionization [14]. Figures $4(\mathrm{~b}, \mathrm{c})$ are photos of the fluorescence spot at the focal point of a $0.85 \mathrm{NA}$ air immersion microscope objective when focusing a laser beam ( $65 \mu \mathrm{J}$ pulse energy). This microplasma has longitudinal and transverse sizes smaller than $30 \mu \mathrm{m}$, observed with an intensified CCD (i-CCD) camera. However, $30 \mu \mathrm{m}$ is not the laser spot size (the vacuum spot size was measured around $1.2 \mu \mathrm{m}$ ), but the size of the fluorescence distribution of the fully expanded plasma. A spot size of $1 \mu \mathrm{m}$ can be achieved with a high NA microscope objective. The plasma spot that contributes to the $\mathrm{THz}$ emission can be a $\mu \mathrm{m}$ or less.

Figure 5 is a schematic illustration for the measurement of the angular dependent $\mathrm{THz}$ emission from a microplasma. The pump arm of the setup

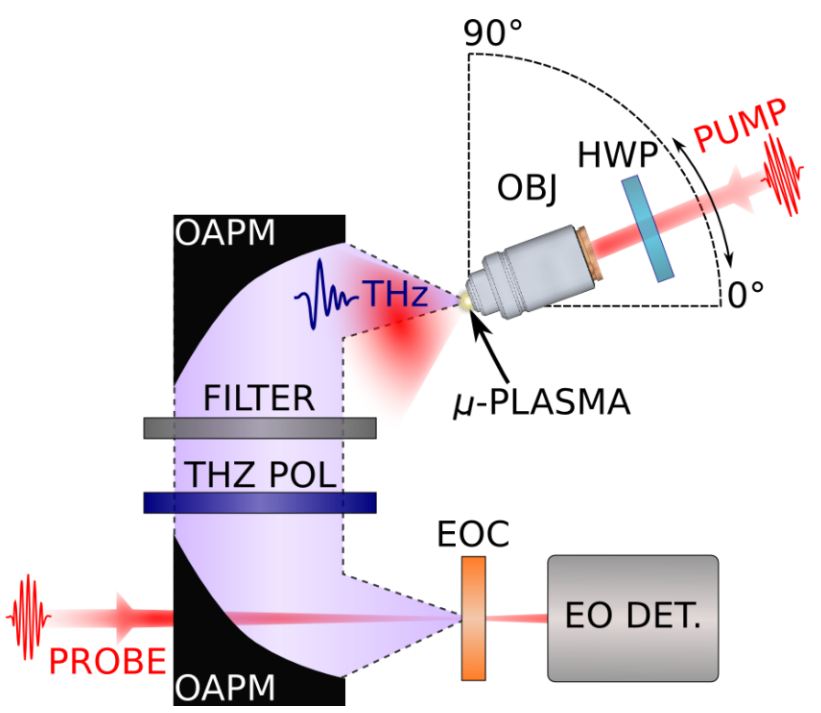

Fig. 5. The experimental setup to implement $\mathrm{THz}$ time-domain spectroscopy. The laser source is a commercial Ti:sapphire amplified laser $(800 \mathrm{~nm}, 100 \mathrm{fs}$, $1 \mathrm{~mJ}, 1 \mathrm{kHz}$ ). The pump beam is focused through a high NA air immersion objective obtaining plasmas with longitudinal and transverse sizes less than $30 \mu \mathrm{m}$. THz radiation is collected and refocused with two off-axis parabolic mirrors. The $\mathrm{THz}$ waveforms are retrieved by free space electro-optical sampling, using a thin $<110>$-cut ZnTe as the detector. A high resistivity silicon wafer is placed in the $\mathrm{THz}$ path in order to prevent the residual pump beam to reach the detector. The pump arm of the setup can be rotated about the geometrical focus of the collecting off-axis parabolic mirror. HWP, half wave plate; OBJ, high NA objective; OAPM, off-axis parabolic mirror; EO, electro-optic crystal; THZ POL, THz polarizer. 
can be rotated about the geometrical focal point of the objective, while the $\mathrm{THz}$ collection optics and detection devices are fixed. Therefore, the spatial radiation pattern of the source can be fully characterized.

In addition to the obvious advantage of reducing the required laser energy, the microplasma offers some unique aspects compared to other $\mathrm{THz}$ sources and sensors. The microplasma can be considered as a freestanding $\mathrm{THz}$ antenna with the subwavelength size $(\sim \lambda / 10)$, with the emission that is spatially separated from the laser excitation (emission direction is perpendicular to the laser propagation direction). Those characteristics might facilitate the coupling and interaction of $\mathrm{THz}$ radiation with microstructures and devices and promote new applications, such as plasmabased $\mathrm{THz}$ near-field techniques.

Moreover, the coherent nature of the emission suggests that more complicated, even arbitrary, radiation patterns might be achieved by combining the radiation from multiple microplasmas, similar to a phased array. Further optimization of the technique, including an improved design of the $\mathrm{THz}$ collection optics, the use of longer excitation laser wavelengths, and laser excitation transverse profile tailoring to maximize the ponderomotive force have the promise to express the full potential of plasma-based $\mathrm{THz}$ techniques with low energy ultrafast lasers.

Figure 6(a) plots the preliminary measurement of $\mathrm{THz}$ time-domain waveforms with a $0.85 \mathrm{NA}$ air immersion objective at different pulse energies. In particular, the red (online) curve represents the smallest $\mathrm{THz}$ waveform we were able to measure in the current experimental condition. The corresponding value of laser pulse energy was $660 \mathrm{~nJ}$, which achieves an improvement of more than one order of magnitude on threshold energy compared to elongated plasma generation geometries. The signal-to-noise ratio can be further improved by using an excitation laser with a higher repetition rate. We expect that by using a Coherent RegA laser with the $250 \mathrm{kHz}$ repetition rate, the pulse energy threshold can be lowered by at least another order of magnitude. In order to realize this technique the use of laser sources with tens of $\mathrm{nJ}$ and about a $1 \mu \mathrm{m}$ focused beam diameter to induce microplasmas is needed. This is a significant task we have to address. (a)

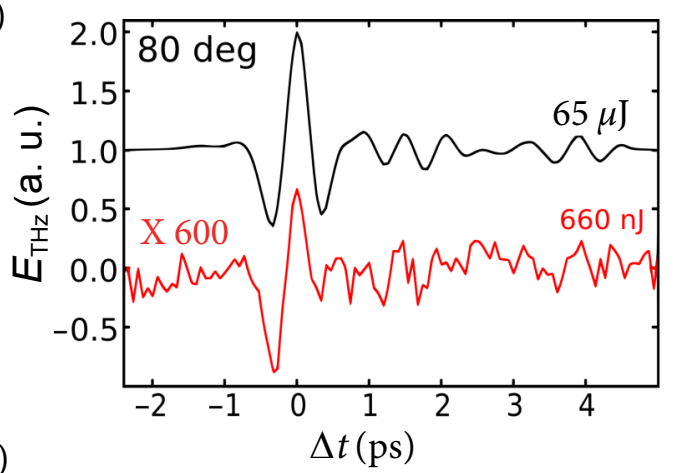

(b)

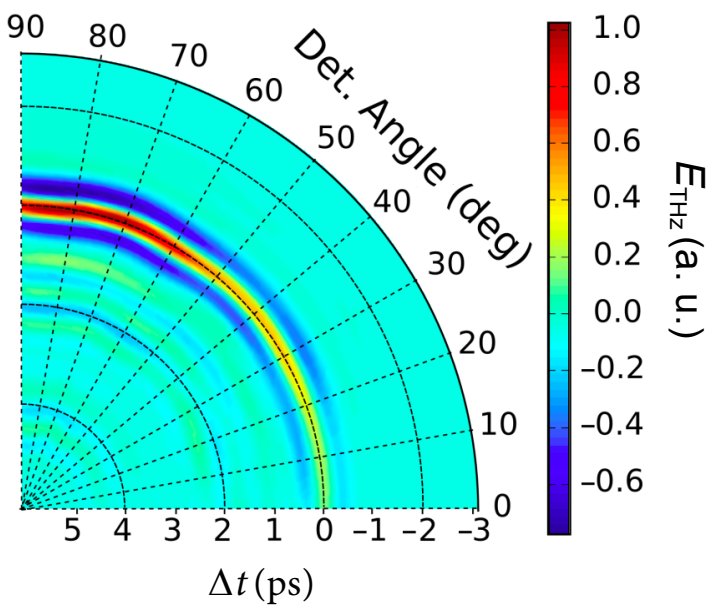

(c)

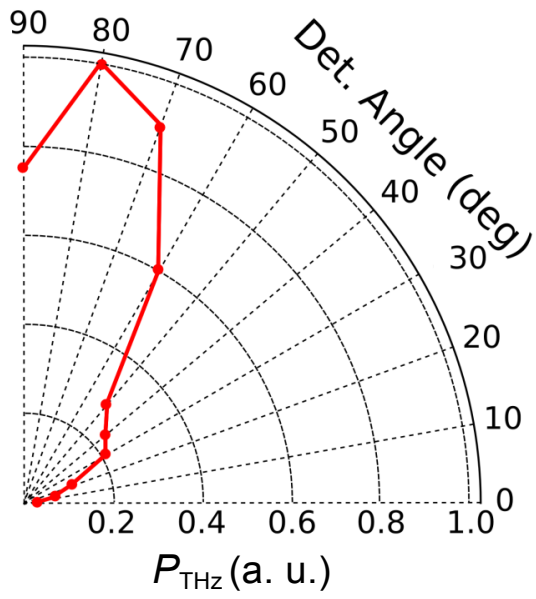

Fig. 6. (a) The THz pulse is generated with a $0.85 \mathrm{NA}$ air immersion objective. The detection angle is $80 \mathrm{de}-$ grees and the pump pulse energies are $65 \mu \mathrm{J}$ (black) and $660 \mathrm{~nJ}$ (red online). (b, c) The measured THz field and power as a function of the detection angle, respectively. The power is calculated as the time integral of the absolute square of the measured $\mathrm{THz}$ waveform.

Figures $6(\mathrm{~b}, \mathrm{c})$ show the temporal $\mathrm{THz}$ waveform and power as a function of the detection angle, which is defined as the angle between the laser propagation direction and the optical axis of the $\mathrm{THz}$ collection optics. Specifically, when 
the detection angle is set at 0 degrees, the system is measuring the forward $\mathrm{THz}$ radiation from the plasma, whereas at 90 degrees the system measures the sideways $\mathrm{THz}$ radiation. The figures show that, with one-colour optical excitation, the $\mathrm{THz}$ emission is at maximum around 80 degrees away from the laser propagation direction.

The characterization of the radiation pattern will enable us to design a better system to collect $\mathrm{THz}$ from the source, allowing to further increase the performance of a $\mathrm{THz}$ air photonics system based on microplasmas. An initial attempt is shown in Figs. $7(\mathrm{a}, \mathrm{b})$, where we demonstrated that by simply using a parabolic reflector we were able to improve the efficiency of the system by a factor of three.

(a)

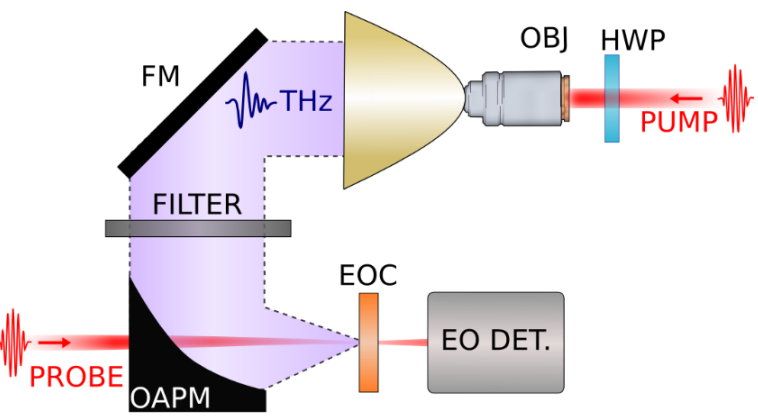

(b)

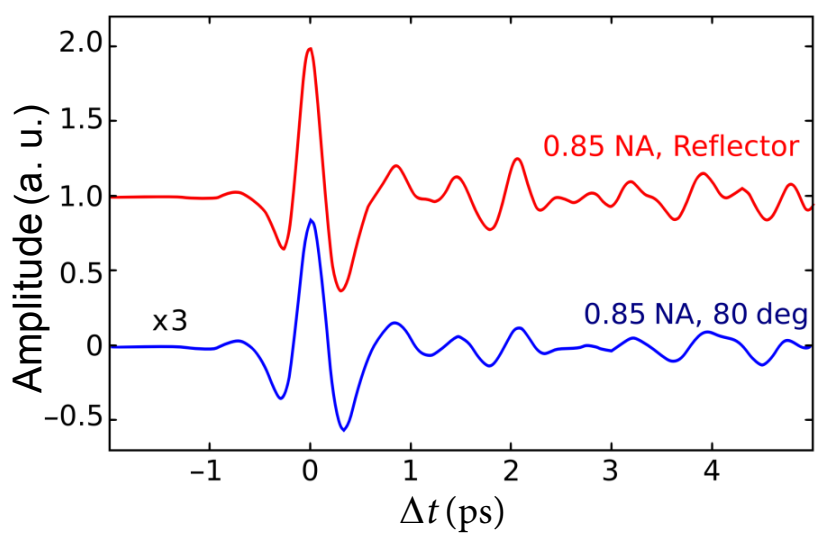

Fig. 7. (a) An experimental setup used to improve the efficiency of the system. The $\mathrm{THz}$ radiated from the microplasma is collimated by a parabolic reflector and detected using electro optics sampling. (b) A comparison between the measured $\mathrm{THz}$ waveforms generated with laser pulse energy of $65 \mu \mathrm{J}$. The trace in red (online) is collected with the parabolic reflector in the setup shown in Fig. 7(a), while the one in blue (online) is measured with the setup shown in Fig. 5 at a detection angle of $80 \mathrm{deg}$.

\section{Ponderomotive force to increase the $\mathrm{THz}$ generation efficiency}

The $\mathrm{THz}$ generation mechanism from microplasma induced by single-colour optical excitation is largely dominated by ponderomotive force. The ponderomotive force is a time average effect depending only on the envelope of the optical pulse and not from the dynamics of single ionization events. The ponderomotive force experienced by a charged particle in an inhomogeneous oscillating electro-magnetic field is expressed by

$$
\mathbf{F}_{\text {Pond }}=-\frac{q^{2}}{4 m \omega^{2}} \nabla|\mathbf{E}|^{2} \text {. }
$$

Here $q$ is the particle charge, $m$ is the mass, and $\mathbf{E}$ is the electric field strength. Its effect is to push charged particles towards areas of low intensity.

In one-colour plasmas, $\mathrm{THz}$ generation is due to the formation of a dipole behind the ionization front of the excitation laser (Fig. 8(a)). Compared

(a)

(b)

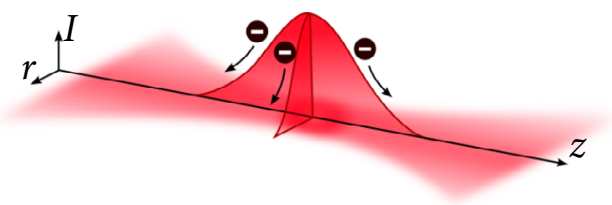

(c)

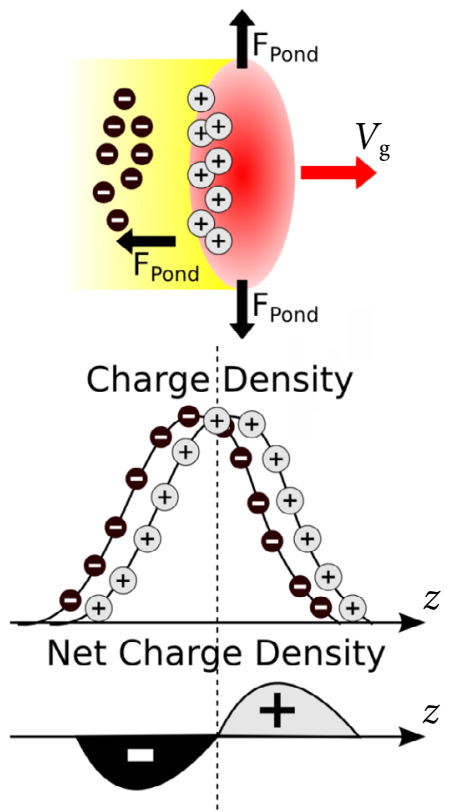

Fig. 8. (a) The laser beam propagates along the $z$ direction, the ponderomotive force created by the gradient of the electric field drives free electrons. (b) Ponderomotive forces drive electrons away in the microplasma, leaving the heavy ions relatively stationary. (c) Net charge density creates an electrical dipole along the $z$ direction. 
to electrons, the ions are many orders of magnitude heavier and can be considered at rest during the ultrafast time scale of the laser pulse. However, the electrons affected by the ponderomotive force are locally accelerated towards the areas of lower intensity, as shown in Fig. 8(b). Over the duration of the laser pulse, many electrons are pushed backward, effectively creating a dipole along the laser propagation direction moving at the group velocity of the femtosecond excitation pulses [14], as shown in Fig. 8(c).

The resulting $\mathrm{THz}$ radiation from this mechanism is a forward propagating cone whose divergence angle increases as the plasma size decreases, as shown in Fig. 9. With the reduction of the plasma length by using lenses with an increasingly shorter focal length, the $\mathrm{THz}$ radiation direction departs from the optical propagation direction, as shown in Fig. 9 .

The following relation between the emitted $\mathrm{THz}$ power from a one-colour plasma and the excitation laser parameters was proposed by Hamster and coworkers in 1994 [15]:

$$
P_{\mathrm{THz}} \propto\left(\frac{W}{R_{0}}\right)^{2}\left(\frac{\lambda}{\tau}\right)^{4} .
$$

Here $W$ is the laser pulse energy, $r_{0}$ is the $1 / \mathrm{e}^{2} \mathrm{ra}$ dius of the laser beam at the focus, $\lambda$ is the laser wavelength, and $\tau$ is the pulse duration. Therefore, Eq. (6) predicts a strong dependence of $\mathrm{THz}$ power on the wavelength and the pulse duration of the excitation laser. The $\mathrm{THz}$ power is proportional to the square of the wavelength, since $R_{0}$ is proportional to $\lambda$. The complete experimental verification of this equation has not been reported yet. However, a Canadian group recently measured a similar wavelength scaling in the case of an elongated two-colour plasma [16]. We propose to use shorter pulse (short $\tau$ ) and longer wavelength (long $\lambda$ ) laser pulse excitation, together with a tight focus (small $R_{0}$ ), to improve the $\mathrm{THz}$ power.

All the experimental observations, including power dependence, polarization, and increased efficiency with higher NA objectives, are consistent with the laser ponderomotive force as the main driver for the $\mathrm{THz}$ transient generation mechanism when a single-colour laser pulse is used.

\section{Microplasma induced by asymmetrical optical excitation}

An asymmetrical optical excitation is obtained by mixing the laser beam fundamental frequency $\omega$ with its second harmonic $2 \omega$. For elongated plasmas it has been demonstrated that the optical-to$\mathrm{THz}$ conversion efficiency increases dramatically by three orders of magnitude when asymmetrical excitation is employed [17].

Unlike the ponderomotive force, the dual-colour laser creates in the plasma a dipole aligned perpendicularly in respect to the optical axis, which therefore leads to $\mathrm{THz}$ emission in the forward direction [18]. It is important to notice that in a plasma generated by dual-colour laser pulses, there is still $\mathrm{THz}$ emission due to the ponderomotive effect, but usually it is much weaker in the case of elongated plasma, due to an order of magnitude lower efficiency compared to the dipole generated by asymmetrical optical excitation. (a)

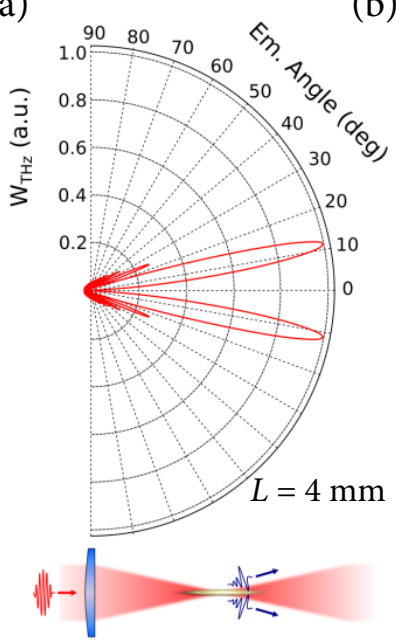

(b)

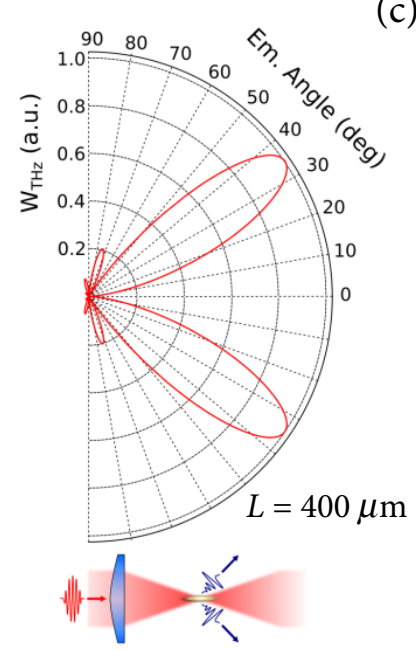

(c)

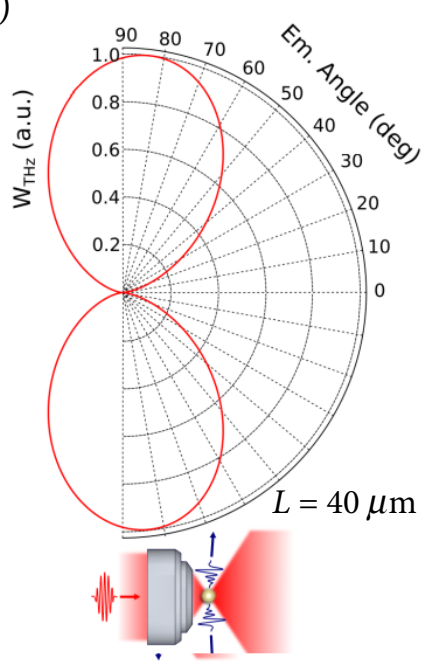

Fig. $9(\mathrm{a}-\mathrm{c})$. The relationship between the $\mathrm{THz}$ emission directions and the plasma length. As the longitudinal size of the plasma decreases, the $\mathrm{THz}$ emission directions move away from the optical axis. In the micro plasma case (c) $\mathrm{THz}$ radiation is emitted nearly perpendicularly with respect to the optical axis. 
Two-colour optical excitation was used to create microplasmas and compare two different physical mechanisms: the ponderomotive force versus the asymmetric optical field. A Schwarzschild objective could be used to combine two colour beams at the focal spot, as shown in Fig. 10. The use of reflective microscope objectives (a classical Schwarzschild design) will enable us to focus the two laser beams without introducing chromatic aberration and dispersion. These objectives are corrected for the thirdorder spherical aberration, coma, and astigmatism, and have negligible higher-order aberrations, resulting in near-diffraction-limited performance.

The Schwarzschild objectives with a $7.8 \mathrm{~mm}$ working distance and $0.50 \mathrm{NA}$ are commercially available. These advantages make them well suited for our dual-colour optical excitation experiment.

It is not clear that the efficiencies of $\mathrm{THz}$ generation due to these two different physical processes compare in the extreme case of the microplasma. In fact, a microplasma allows the study of both mechanisms at the same time. This can be understood by considering that the ponderomotive force and the asymmetric optical field will lead to $\mathrm{THz}$ emission in different directions, sideways and forward, respectively. Moreover, $\mathrm{THz}$ emission from the asymmetric field can be coherently controlled by changing the phase between $\omega$ and $2 \omega$ : it can even be totally suppressed [19]. Therefore, the contribution to the $\mathrm{THz}$ emission from both mechanisms can be spatially separated and verified.

By combining lateral emission due to the ponderomotive force and the forward emission due to the asymmetrical optical excitation, we also anticipate the possibility to realize a $\mathrm{THz}$ quasilambertian source (the emitted $\mathrm{THz}$ radiation pattern would be close to a semi-sphere). This kind of source can be used for metrology applications, such as the characterization of $\mathrm{THz}$ antennas and receivers. We expect this kind of source to have a significant impact in the rapidly growing area of THz communications.

\section{Microplasma with resonant gases}

In addition to the tightly focused optical beam with controlled polarization, we also plan to use different gases to form microplasmas. In particular, we are interested in studying gases with a low ionization potential, therefore requiring less laser energy to form a plasma. Examples of such gases are cesium (Cs) or rubidium ( $\mathrm{Rb}$ ) vapour, with ionization potentials of 3.89 and $4.17 \mathrm{eV}$, respectively, much lower than the values of molecular nitrogen, $15.6 \mathrm{eV}$, and oxygen, $12.1 \mathrm{eV}$. The Keldysh-Perelomov ionization theory also indicates their ionization rate is about five times higher than that of nitrogen [20]. Therefore, those gases are predicted to require much less laser energy and be a more efficient $\mathrm{THz}$ source in the microplasma regime, as compared to ambient air. Moreover, cesium and rubidium have strong optical absorption lines, i.e. resonances, in the Ti:sapphire laser frequency range. These strong absorptions are expected to provide interesting physics effects in the $\mathrm{THz}$ emission, such as a resonant behaviour and perhaps improved optical-to-THz conversion efficiency.

\section{THz detection using microplasmas}

$\mathrm{THz}$ detection schemes based on $\mathrm{THz}$ air photonics, such as $\mathrm{THz}$ radiation-enhanced-emissionof-fluorescence (REEF) [22-23] and $\mathrm{THz}$ air-biased-coherent-detection (ABCD) [4], have shown the possibility to achieve ultra broadband and sensitive detection covering the whole $\mathrm{THz}$ gap, 0.1 to $10 \mathrm{THz}$. We propose to study the implementation of those schemes using laser-induced microplasmas, in



Fig. 10. The concept of dual-colour (online) microplasma. The fundamental, $\omega$, and the second harmonic beams, $2 \omega$, are tightly focused with a high-NA reflective objective. 
order to enable their utilization with low energy ultrafast sources.

The main practical challenge is the short working distance of common microscope objectives. This restricts the $\mathrm{THz}$ beam to be measured with the laser probe beam in the usual co-propagation geometry, where both beams propagate in the same direction. However, the theory for both detection techniques does not forbid their implementation in non-collinear geometries and also in the counter-propagation geometry, where the two beams are collinear but propagate in opposite directions. We plan to study whether the counter propagation geometry is experimentally feasible for both $\mathrm{THz}-$ REEF and THz-ABCD.

In THz-REEF, the plasma fluorescence is enhanced by the presence of the $\mathrm{THz}$ electric field. By measuring the change in fluorescence, the $\mathrm{THz}$ waveform can be retrieved. The technique can also be implemented in counter-propagation, as shown in Fig. 11(a). The THz-ABCD has been discussed in Section 2. Figure 11(b) illustrates how to realize it using a microplasma. In this configuration we propose to collect the $\mathrm{THz}$ field-induced second harmonic, directly with the objective used to cre-

(a)

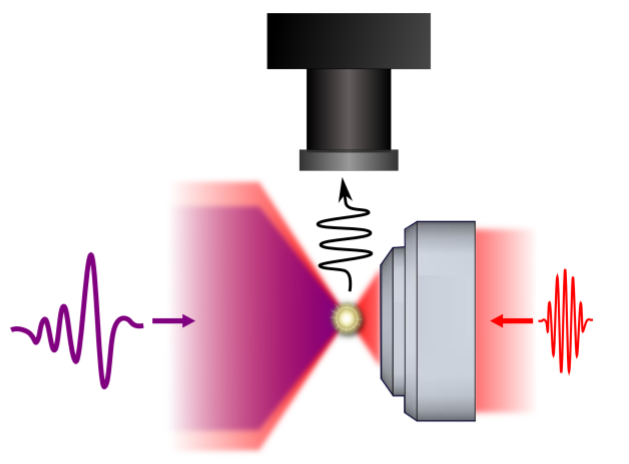

(b)

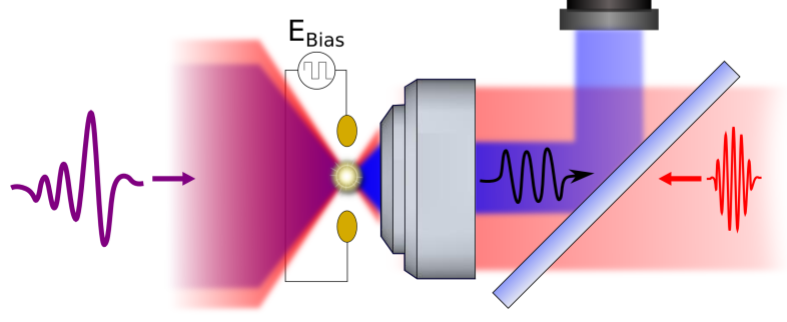

Fig. 11. Conceptual implementation in the counter propagation geometry of THz-REEF (a) and $\mathrm{THz}-$ $\mathrm{ABCD}(\mathrm{b})$ using laser-induced microplasmas. ate the microplasma. The second harmonic is going to be directed toward a photo multiplier tube with a dichroic mirror. A pair of electrodes will provide the local oscillator bias in order to achieve coherent detection.

\section{Microplasmas arrays and tailoring the ponderomotive force}

High numerical focusing of laser beams has been used to demonstrate breakthrough phenomena such as optical trapping [24], optical levitation [25], and optical lift [26]. Some of these techniques rely on the precise control and shaping of the spatial intensity distribution of the laser beam at the focal plane of the microscope objective. This has allowed the development of the so-called "optical tweezer", where the laser beam can be used to "pick", "move" and "release" particles of sizes up to few microns in diameter.

Recently, the development of commercially available and user-friendly spatial light modulators has helped to improve the degree of control of the intensity pattern at the focal plane. This allows the realization of multiple optical tweezers, which are able to operate at the same time and independently, as shown in Fig. 12. We plan to use a spatial light modulator to tailor the in-

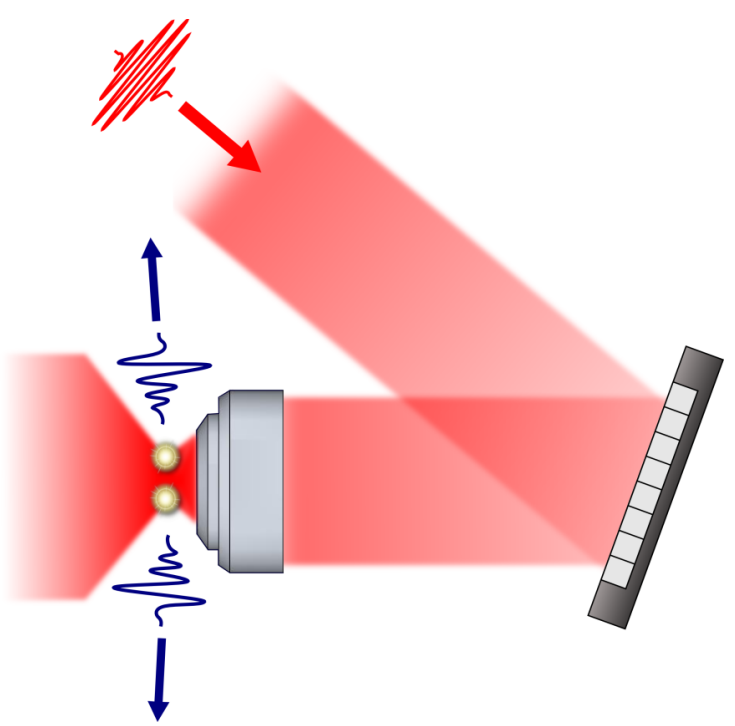

Fig. 12. Generation of multiple microplasmas is achieved by applying a phase mask to the laser excitation through a reflective spatial light modulator. By controlling the distance and the phase relations between the sources it is possible to control the $\mathrm{THz}$ emission pattern. 
tensity distribution of the laser beam close to the focal plane of the objective. By doing so it is possible to manipulate the effect of the ponderomotive force on the free electrons with the goal of increasing the emission efficiency of the process and also to gain some control on the radiation pattern of the source. We indeed expect to be able to arbitrarily change the main emission direction by generating multiple microplasmas. This will be achieved by carefully choosing the distance and the phase relation between the sources, similarly to a linear phase array.

\section{Microplasmas for $\mathrm{THz}$ coupling with micro- and nano-structures}

The microplasma has a sub-wavelength size compared to the emitted $\mathrm{THz}$ frequency. Alternatives to obtain sub-wavelength $\mathrm{THz}$ sources are to tightly focus the excitation laser onto a thin nonlinear crystal (thickness less of $20 \mu \mathrm{m}$ ) or to place a metal shield with a sub-wavelength pinhole in front of an extended THz source [27]. Both methods have drawbacks compared to the microplasma. In fact, a very thin crystal is not mechanically stable. It is easy to break, and under a tight focusing condition can be easily damaged even by a very low energy power excitation. Moreover, the $\mathrm{THz}$ low spatial frequencies are totally internally reflected at the crystal-air interface due to their high divergence angle. In the pinhole approach, only a little portion of $\mathrm{THz}$ power is transmitted through the aperture. Furthermore, the power throughput scales with the sixth power of the pinhole diameter, making this technique very inefficient. In both cases the strength of $\mathrm{THz}$ field obtainable is very limited.

Unlike the thin nonlinear crystal, the microplasma does not have a damage threshold and does not suffer from the problem of total internal reflection, since the refractive index interface with the surrounding air is small and gradual. In opposition to the pinhole, the $\mathrm{THz}$ power is expected to grow when the size of the source is reduced (see Eq. (2)). The abovementioned reasons make the microplasma attractive for the free space coupling of intense $\mathrm{THz}$ with structures that are small compared to its wavelength. Examples of such structures are nanoprobes, tapered waveguides, and micro- and nano-antennas, shown in Fig. 13.

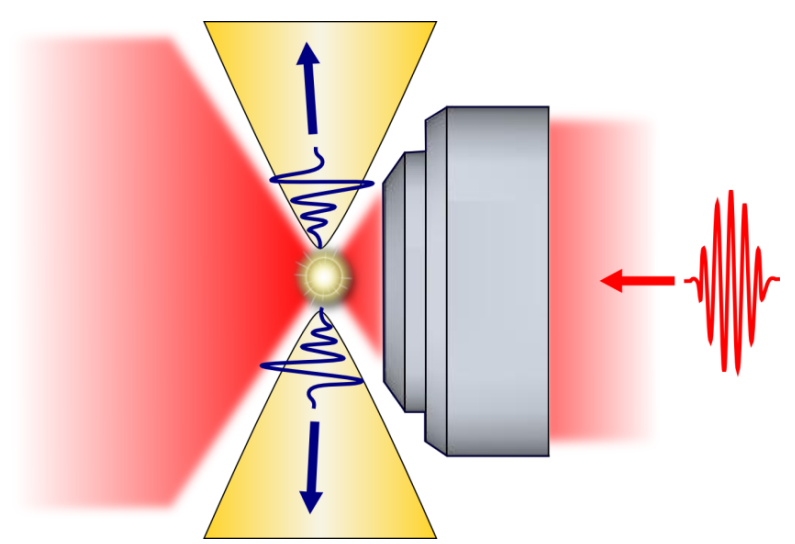

Fig. 13. THz generated from a microplasma can couple in the near field of the source devices much smaller than the $\mathrm{THz}$ wavelength. As a concept, we show the coupling of the $\mathrm{THz}$ wave with a bow tie antenna (a triangle pattern, yellow online).

\section{Basic science: electron dynamics, approaching sub-micron scale and beyond}

One of fundamental basic scientific tasks in this proposal is to use $\mathrm{THz}$ emission from the plasma to study/probe electron dynamics. The laser-induced microplasma is a suitable platform for that. In fact, unlike an elongated plasma, in a microplasma the laser intensity and the electron density are not clamped. Therefore, in a single experiment one can change the electron density by several orders of magnitude, accessing the two limiting cases of weak collisionless and dense collisional plasmas $\left(>10^{19} \mathrm{~cm}^{-3}\right)$.

The plasma density has not been measured yet. When we used $65 \mu \mathrm{J}$ pulse energy and focused the beam with a microscope objective ( $0.85 \mathrm{NA})$, the estimated electron densities were as high as $10^{19} \mathrm{~cm}^{-3}$ at the centre of the plasma. With the full laser power near the $\mathrm{mJ}$ range, a full ionization state can be achieved. Further degrees of freedom for controlling the ionization can be obtained using a dual-colour beam as the laser excitation.

Using a full aperture high NA objective and a short wavelength laser, a sub-micron focal spot can be achieved. The $\mathrm{THz}$ wave is emitted right after the gas ionizes and possibly well before the fluorescence appears. Even if the time integrated fluorescence spot is measured to be about tens of microns by an i-CCD camera, the actual plasma spot at the time of $\mathrm{THz}$ emission should be close to the diffraction limit ( $\mu \mathrm{m}$ or less). 
The fundamental basic research is to focus on the extreme case of $\mathrm{THz}$ emission from the micro-nano scale to the scale of molecule clusters or even of single atoms/molecules, so to really isolate the physics of emission and retrieve information regarding the behaviour of ionized single molecules. Our proposed research of micro-nano plasma could serve as a stepping-stone to lead a new fundamental basic research: $\mathrm{THz}$ wave emission and detection with single atoms/molecules.

\section{Challenge and opportunities}

One of the biggest challenges for real world application is that a filament formation needs high peak laser intensities, which are usually obtained with an amplified laser system. Such a source is expensive and requires frequent maintenance from a skilled technician. For this reason, one of the most popular laser sources is the oscillator. In fact, the total number of laser oscillators with $\mathrm{nJ}$ pulse energy worldwide is more than one order of magnitude higher than the total number of amplified lasers $(\mathrm{mJ})$. Our survey shows that the total number of oscillators ( $\mathrm{nJ}$ ) and amplified lasers provided by three laser companies is about 14,000 and 2,100 , respectively. The average cost of an oscillator is about $1 / 4$ to $1 / 2$ of the amplified laser.

The use of oscillator lasers in $\mathrm{THz}$ air photonics has been hindered because they do not allow for the generation of the typical elongated plasmas currently used. However, a simple estimation shows the feasibility for an oscillator to generate a microplasma. Ambient air is ionized at laser peak intensity values of $10^{12} \sim 10^{13} \mathrm{~W} / \mathrm{cm}^{2}$. For our calculation, we can consider an ultrafast oscillator with the following parameters: $1 \mathrm{~W}$ average power, $10 \mathrm{fs}$ pulse duration, and $80 \mathrm{MHz}$ repetition rate. The pulse energy for such laser source is $12.5 \mathrm{~nJ}$, corresponding to a peak power of $1.2 \mathrm{MW}$, assuming a Gaussian profile. If we focus this source to a diameter of $1 \mu \mathrm{m}$, we are able to obtain a peak intensity of $10^{14} \mathrm{~W} / \mathrm{cm}^{2}$. For this value of intensity ambient air can be ionized and $\mathrm{THz}$ pulse will be emitted.

The main difficulty is to efficiently collect the emitted $\mathrm{THz}$ radiation from the source and detect it with a high signal-to-noise ratio (SNR). To our advantage, a standard laser oscillator has a much higher repetition rate $\left(10^{8}\right)$ than that of a typical amplified laser $\left(10^{3}\right)$. This corresponds to an increase of the sampling rate for the detected signal of a factor of $10^{5}$, which will result in improvement by a factor of 300 of the SNR at parity of the detected signal strength because the SNR is proportional to the square root of the repetition rate using the sampling technology. We believe that the increase in the repetition rate is able to compensate for the signal strength reduction due to a low energy excitation source.

There are several commercially available $\mathrm{THz}$ systems using the photoconductive or electro-optic $\mathrm{THz}$ wave emitters and sensors. Each of them has their own pros and cons with respect to power, bandwidth, polarization, and cost. The unique features of $\mathrm{THz}$ emitters and sensors based on microplasma are the extremely low laser energy requirement (plasmas can be generated from commercially available fibre lasers used for optical communication) for $\mathrm{THz}$ air photonics, ultrabroad bandwidth, ultra-flat spectrum, and ease of operation.

The microplasma source is far less efficient compared to solid-state sources. The efficiency with microplasma is around $10^{-5}-10^{-6}$, while with antennas and solid states at low energy the efficiency is $10^{-2}-10^{-3}$. Therefore, we do not see microplasma as a replacement for standard solid-state sources or low energy laser sources. However, microplasma is a source with unique features that are not found in any other $\mathrm{THz}$ sources, including: (i) sideways radiation pattern; (ii) freestanding system geometry; (iii) generated in air; (iv) sub-wavelength size. Microplasma is probably able to generate the highest $\mathrm{THz}$ fields of any subwavelength $\mathrm{THz}$ source. Usually a sub-wavelength $\mathrm{THz}$ source is obtained by focusing a laser on a very thin nonlinear crystal. To make the source smaller, the focus must be tighter, and therefore the laser energy must be kept to a bare minimum in order to avoid damaging the crystal. Microplasma does not have this problem. Also, coupling the $\mathrm{THz}$ from the microplasma to the microstructure should be easier as there is no interface.

$\mathrm{THz}$ air plasma may not be a universal solution for all the challenging problems in $\mathrm{THz}$ air photonics. Other limitations are generation efficiency, collection efficiency, and polarization management. However, these challenges provide opportunities to investigate and to expand our knowledge 
of the physical processes involved. The use of a mode-locked fibre laser at eye safe communication wavelength range helps a lot in terms of safety, especially in comparison with typical Ti:sapphire amplified lasers. We must investigate a feasible method for a mini $\mathrm{THz}$ air photonics system using microplasma, which was previously considered to be impossible.

\section{Concluding remarks}

Our estimation indicates that the microplasma approach may reduce the required optical pulse energy by five orders of magnitude, while still obtaining a comparable or better signal-to-noise ratio for $\mathrm{THz}$ time-domain spectroscopy. This would utilize the full potential of $\mathrm{THz}$ air plasma science at low excitation regimes, leading to greatly improved and increasingly affordable $\mathrm{THz}$ instruments. The $\mathrm{THz}$ microplasma project will lead to key technologies that will enable further interdisciplinary research and the continued advancements of numerous $\mathrm{THz}$ wave sensing and spectroscopy developments.

The scientific investigation of $\mathrm{THz}$ microplasmas is a high-risk project. If successful, it will lead to substantial advancements in next-generation $\mathrm{THz}$ sources and system integrations in nanoplasma and "a single molecule". In particular, the microplasma is a convenient platform to optimize the protocols and technologies to eventually measure the $\mathrm{THz}$ radiation emission and interaction with "a single molecule".

\section{Acknowledgements}

This research was sponsored by the Army Research Office and was accomplished under Grant No. US ARMY W911NF-17-1-0428. The data of microplasma with resonance gases was taken by Dr. Quan Sun.

\section{References}

[1] A. Couairon and A. Mysyrowicz, Femtosecond filamentation in transparent media, Phys. Rep. 441, 47-189 (2007).

[2] N. Karpowicz, J. Dai, X. Lu, Y. Chen, M. Yamaguchi, H. Zhao, X.-C. Zhang, L. Zhang, C. Zhang,
M. Price-Gallagher, C. Fletcher, O. Mamer, A. Lesimple, and K. Johnson, Coherent heterodyne time-domain spectrometry covering the entire “terahertz gap", Appl. Phys. Lett. 92, 011131 (2008).

[3] X. Xie, J. Dai, and X.-C. Zhang, Coherent control of $\mathrm{THz}$ wave generation in ambient air, Phys. Rev. Lett. 96, 075005 (2006).

[4] J. Dai, X. Xie, and X.-C. Zhang, Detection of broadband terahertz waves with a laser-induced plasma in gases, Phys. Rev. Lett. 97, 103903 (2006).

[5] D.J. Cook and R.M. Hochstrasser, Intense terahertz pulses by four-wave rectification in air, Opt. Lett., 25, 1210-1212 (2000).

[6] S. Tzortzakis, G. Méchain, G. Patalano, Y.-B. André, B. Prade, M. Franco, A. Mysyrowicz, J.-M. Munier, M. Gheudin, G. Beaudin, and P. Encrenaz, Coherent subterahertz radiation from femtosecond infrared filaments in air, Opt. Lett. 27, 19441946 (2002).

[7] M. Kress, T. Löffler, S. Eden, M. Thomson, and H.G. Roskos, Terahertz-pulse generation by photoionization of air with laser pulses composed of both fundamental and second-harmonic waves, Opt. Lett., 29, 1120-1122 (2004)

[8] T. Löffler, M. Kress, M. Thomson, and H.G. Roskos, Efficient terahertz pulse generation in laserinduced gas plasmas, Acta Phys. Pol. A, 107, 99 (2005).

[9] T. Bartel, P. Gaal, K. Reimann, M. Woerner, and T. Elsaesser, Generation of single-cycle THz transients with high electric-field amplitudes, Opt. Lett. 30, 2805-2807 (2005).

[10]A. Nahata and T.F. Heinz, Detection of freely propagating terahertz radiation by use of optical second-harmonic generation, Opt. Lett. 23, 67-69 (1998).

[11]D.J. Cook, J.X. Chen, E.A. Morlino, and R.M. Hochstrasser, Terahertz-field-induced second-harmonic generation measurements of liquid dynamics, Chem. Phys. Lett. 309, 221-228 (1999).

[12]R.W. Boyd, Nonlinear Optics (Academic Press, Boston, 1992).

[13]H. Hamster, A. Sullivan, S. Gordon, W. White, and R.W. Falcone, Subpicosecond electromagnetic 
pulses from intense laser-plasma interaction, Phys. Rev. Lett. 71, 2725-2728 (1993).

[14]C. D’Amico, A. Houard, M. Franco, B. Prade, and A. Mysyrowicz, Conical forward $\mathrm{THz}$ emission from femtosecond-laser-beam filamentation in air, Phys. Rev. Lett., 98, 235002 (2007).

[15]H. Hamster, A. Sullivan, S. Gordon, and R.W. Falcone, Short-pulse terahertz radiation from highintensity-laser-produced plasmas, Phys. Rev. E 49, 671-678 (1994).

[16]M. Clerici, M. Peccianti, B. Schmidt, L. Caspani, M. Shalaby, M. Giguere, A. Lotti, A. Couairon, F. Legare, T. Ozaki, D. Faccio, and R. Morandotti, Wavelength scaling of terahertz generation by gas ionization, Phys. Rev. Lett. 110, 253901 (2013).

[17] K.Y. Kim, J.H. Glownia, A.J. Taylor, and G. Rodriguez, Terahertz emission from ultrafast ionizing air in symmetry-broken laser fields, Opt. Express 15, 4577-4584 (2007).

[18]J. Dai, N. Karpowicz, and X.-C. Zhang, Coherent polarization control of terahertz waves generated from two-colour laser-induced gas plasma, Phys. Rev. Lett. 103, 023001 (2009).

[19]H. Wen and A.M. Lindenberg, Coherent terahertz polarization control through manipulation of electron trajectories, Phys. Rev. Lett. 103, 023904 (2009).

[20]X. Suan and X.-C. Zhang, Terahertz radiation in alkali vapour plasmas, Appl. Phys. Lett. 104, 191106 (2014).
[21]L. Neukirch, J. Gieseler, R. Quidant, L. Novotny, and A. Vamivakas, Observation of nitrogen vacancy photoluminescence from an optically levitated nanodiamond, Opt. Lett. 38, 2976-2979 (2013).

[22]J. Liu and X.-C. Zhang, Terahertz-radiationenhanced emission of fluorescence from gas plasma, Phys. Rev. Lett. 103, 235002 (2009).

[23]J. Liu, J. Dai, S.L. Chin, and X.-C. Zhang, Broadband terahertz wave remote sensing using coherent manipulation of fluorescence from asymmetrically ionized gases, Nat. Photonics 4, 627-631 (2010).

[24]A. Ashkin, J. Dziedzic, J. Bjorkholm, and S. Chu, Observation of a single-beam gradient optical trap for dielectric particles, Opt. Lett. 11, 288290 (1986).

[25]A. Ashkin and J. Dziedzic, Optical levitation by radiation pressure, Appl. Phys. Lett. 19, 283-285 (1971).

[26]G. Swartzlander, T. Peterson, A. Artusio-Glimpse, and A. Raisanen, Stable optical lift, Nat. Photonics 5, 48-51 (2011).

[27]J. Liesener, M. Reicherter, T. Haist, and H. Tiziani, Multi-functional optical tweezers using computer-generated holograms, Opt. Commun. 185, 77-82 (2000).

[28]A. Adam, Review of near-field terahertz measurement methods and their applications, J. Infrared Millim. Terahertz Waves 32, 976-1019 (2011).

\title{
TERAHERCINĖ MIKROPLAZMOS FOTONIKA IR DAR DAUGIAU
}

\author{
X.-C. Zhang ${ }^{\text {a,b }}$, F. Buccheri ${ }^{\text {a }}$ \\ ${ }^{a}$ Ročesterio universiteto Optikos institutas, Ročesteris, JAV \\ ${ }^{\mathrm{b}}$ Capital Normal universitetas, Pekinas, Kinija
}

